\title{
An Evaluation of Combustion Kinetics for the Synthesis Reaction of the Reinforcing Phase During Casting
}

\author{
Tomasz Wiktor, Sebastian Sobula* $^{*}$, Andriy Burbelko $\oplus_{\odot}$, Michał Ptasznik \\ AGH University of Science and Technology, Faculty of Foundry Engineering, Reymonta St. 23, 30-059 Krakow, Poland \\ *e-mail: sobula@agh.edu.pl
}

\begin{abstract}
(C) 2020 Authors. This is an open access publication, which can be used, distributed and reproduced in any medium
\end{abstract} according to the Creative Commons CC-BY 4.0 License requiring that the original work has been properly cited.

Received: 30 December 2019/Accepted: 19 June 2020/ Published online: 30 June 2020

This article is published with open access at AGH University of Science and Technology Press

\begin{abstract}
The computer modeling of the solidification process in castings with local composite reinforcement (LCR) obtained as a result of in situ reactions of self-propagating high temperature synthesis (SHS) is difficult due to limited data on the thermo-physical parameters of exothermic effects and the kinetics of the synthesis reaction. In the present study, Hadfield cast steel casting was manufactured with LCR containing titanium carbide particles obtained in situ by the SHS method. Reaction kinetics of titanium carbide synthesis in the composite casting were determined on the basis of temperature measurements in the area of LCR during the process. For the estimation of the reaction, the Fourier Thermal Analysis method was used. The paper presents the results of temperature measurement and the results of the calculation of SHS reaction kinetics. It was found that the reaction time under the conditions of the analyzed casting is below $3 \mathrm{~s}$.
\end{abstract}

\section{Keywords:}

MMC, SHS reaction kinetics, Fourier thermal analysis, computer modelling

\section{INTRODUCTION}

The creation of local composite reinforcements (LCR) zones with increased hardness and resistance to abrasion allows the extension of the working time of iron alloy castings. One of the most well-known methods of achieving this effect is the synthesis of the reinforcing phases as a product of an exothermic, chemical reaction occurring in situ in the selected areas of the casting. Reinforcing particles are formed during the reaction of the Self-Propagating High-Temperature Synthesis process (SHS) in a component composed of compressed powders containing synthesis reaction reagents attached to the mold cavity.

The manufacturing and replacement of the ordinary cast parts with cast components with local composite zones is one of the important ways of the evolution of modern casting. Composite castings are characterized by the high hardness of the local composite reinforcements (LCR), which directly influences the lifetime of these castings. One popular method to obtain LCR is by using in situ SHS processes during casting. This method makes it possible to obtain very hard localized composite zones reinforced with carbide particles. Correct selection of raw material composition for SHS reaction provides a hardness of reinforced sections at the level from HV650 to HV900 [1, 2].
The composition of the raw materials for the manufacturing of local composite zones includes both active components (powders of the carbide-forming elements, such as titanium, tungsten, molybdenum and carbon powder) and a moderator. The moderator is used to improve dimensional stability and control of SHS reaction. The chemical composition of the moderator is usually similar to the base alloy chemical composition [3].

The energy which is released during the exothermic synthesis of reinforced particles in areas of LCR influences the temperature field in casting and in the mold. The thermal effect of synthesis depends on the weight fraction of reagents and the moderator in compact. The control of the moderator weight fraction is a fundamental problem during LCR synthesis in castings.

If the amount of active ingredients is small and the amount of moderator is large, the SHS reaction can be terminated before completion, due to the small amount of heat generated by the synthesis. This results in low infiltration, poor bonding between the base alloy and LCR, and finally low properties in the local area of the composite. Otherwise, when the weight fraction of the reagent is relatively large, the amount of heat released as a result of the reaction to the surrounding volume causes the formation of coarse reinforcement particles, which are dispersed in the volume of the casting. 
The use of an excessive amount of active ingredients means that the synthesis temperature may clearly exceed the initial temperature of the liquid steel. Such an example is shown in Figure 1: the changes of temperature are presented in the SHS reaction zone (LCR1) and in the base carbon cast steel casting (L25GS, i.e. according to PN standard) at the distance of $10 \mathrm{~mm}$ from surface of compact. In the cited experiment, green compact was made from titanium and carbon powders in stoichiometric ratio. A high rate of temperature increase is visible after the beginning of the synthesis reaction in the component containing substrates for the formation of titanium carbide. Additionally, this heat affects solidification and leads to the formation of hot spots in the local area of the composite.

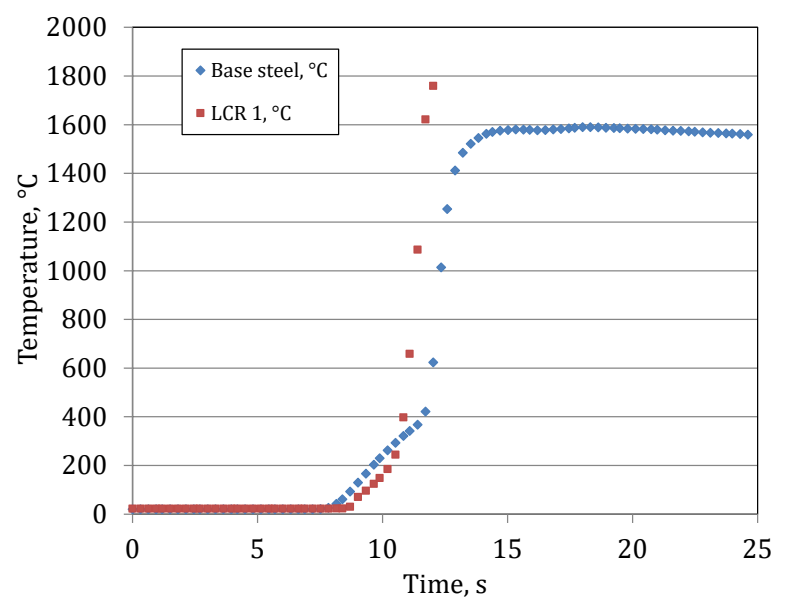

Fig. 1. Temperature changes in steel casting and in the SHS reaction zone [4]

A significant amount of thermal energy introduced in these places into the casting must be taken into account when developing the technology for producing such castings. Designing the technological process of the production of LCR castings encounters difficulties related to limited access to thermo-physical data and data concerning the kinetics of the fusion reaction of reinforcing phases. Problems related to the measurement and evaluation of exothermic material parameters in laboratory and industrial conditions are presented in [5-7].

The use of computer-aided analysis of cooling curves is known in foundry practice for measuring of exothermic transformation kinetics. The Fourier-Thermal Analysis (FTA) method makes it possible to estimate the intensity of exothermic processes occurring in the local volume of the analyzed material. The intensity of the thermal effect using FTA is assessed by comparing the rate of temperature change with a base line calculated from the Laplace operator of temperature.

The fundamentals of the Fourier Thermal Analysis (FTA) method has been presented in $[8,9]$. In these papers, FTA has been used to determine the kinetics of gray cast iron solidification in the local region. Later, the FTA method has been successfully used to characterize the solidification process for various metal alloys, such as: aluminum-based materials [10-15], magnesium alloy [16], different $\mathrm{Pb}-\mathrm{Sb}$ alloys [17], and for cast-composites [18]. As it has been proven in [19], the FTA method can also be used to analyze the kinetics of thermal decomposition of resin binders intended for use in molding sands.

In this paper, the FTA method was used to assess the thermal effect of an exothermic SHS reaction in the LCR area in steel castings.

\section{THEORETICAL BASIS}

A non-steady state temperature field in the medium with an exothermic or endothermic reaction can be described by the Fourier equation which includes a heat source:

$$
\frac{\partial T}{\partial t}=a \nabla^{2} T+\frac{\dot{Q}}{c}
$$

where:

$$
\begin{aligned}
\partial T / \partial t & - \text { the rate of material cooling, } \mathrm{K} / \mathrm{s}, \\
a & - \text { the thermal diffusivity, } \mathrm{m}^{2} / \mathrm{s}, \\
\dot{Q} & - \text { volumetric heat generated or absorbed by the } \\
& \quad \text { substance matter during the reaction, } \mathrm{W} / \mathrm{m}^{3}, \\
c & - \text { the volumetric specific heat, } \mathrm{J} /\left(\mathrm{m}^{3} \mathrm{~K}\right), \\
\nabla^{2} & - \text { the Laplace operator }\left(\nabla^{2}=\partial^{2} / \partial x+\partial^{2} / \partial y+\partial^{2} / \partial z\right), \\
T & - \text { temperature, } \mathrm{K} .
\end{aligned}
$$

For the estimation of heat generation rate, at the first stage of FTA the apparent value of the temperature diffusivity of the substance $\left(a_{\mathrm{av}}, \mathrm{m}^{2} / \mathrm{s}\right)$ should be determined on the basis of the temperature measurement at several points of the analyzed sample. This value is determined by dividing the rate of temperature changes by Laplacian of the temperature $\nabla^{2} T$ :

$$
a_{\mathrm{av}}=\frac{\frac{\partial T}{\partial t}}{\nabla^{2} T}
$$

Outside the reaction zone, the apparent value of temperature diffusivity $\left(a_{\mathrm{av}}, \mathrm{m}^{2} / \mathrm{s}\right)$ is slightly dependent on the temperature. In the reaction zone, the changes are intense and irregular.

At the second stage of FTA, the interpolated value of the temperature diffusivity $a_{\text {int }}$ should be estimated for the reaction period on the basis of the temperature diffusivity levels of the analyzed substance before $\left(a_{1}\right)$ and after $\left(a_{2}\right)$ the exothermic reaction. For precise analysis $a_{1}$ and $a_{2}$ values can be measured in laboratory conditions. In the case of approximate calculation, these parameters can be determined graphically from " $a_{\mathrm{av}}$-time" diagram at the beginning and end of the range of irregular changes of the apparent temperature diffusivity.

Heat generation rate can be estimated based on the results of measurements using the equation:

$$
\dot{q}=\frac{\dot{Q}}{c}=\left(a_{\mathrm{av}}-a_{\mathrm{int}}\right) \cdot \nabla^{2} T
$$

For the measurements, the temperature-time derivative and second spatial temperature derivative are replaced in the Equations (2) and (3) by proper central differential quotients. 
Volumetric heat released as a result of the exothermic reaction should be calculated by temporal integration between the time instants of the reaction start $\left(t_{1}\right)$ to reaction finish $\left(t_{2}\right)$ :

$$
\Delta H=\int_{t_{1}}^{t_{2}} \dot{q}(\tau) \cdot c \cdot d \tau
$$

Let as assume that changes of the volumetric specific heat of the analyzed substance with respect to the average value $\bar{c}$ are small during the reaction period. Under such conditions:

$$
\frac{\Delta H}{\bar{c}} \approx \int_{t_{1}}^{t_{2}} \dot{q}(\tau) \cdot d \tau
$$

and $\dot{q}$ value (measured by $\mathrm{K} / \mathrm{s}$ ) may be used for the indication of the reaction rate. In this case, the conversion level of the analyzed exothermic reaction $f$ may be calculated by the numerical integration of this value by time in the following way:

$$
f(t)=\frac{\int_{t_{1}}^{t} \dot{q}(\tau) d \tau}{\frac{\Delta H}{\bar{c}}}
$$

where $t_{1}$ and $t_{2}$ mean the time instants of the reaction beginning and end.

Extent of the SHS reaction can be calculated on the basis of Equations (5) and (6) as:

$$
f(t)=\frac{\int_{t_{1}}^{t} \dot{q}(\tau) d \tau}{\int_{t_{1}}^{t_{2}} \dot{q}(\tau) d \tau}
$$

\section{EXPERIMENTAL PART}

In the experimental part, measurements of the temperature field were carried out. The scheme of experimental casting is presented in Figure 2. The base casting was made of Hadfield cast steel (grade GX-120Mn13 according to PN-EN 10349:2009 standard). The compact, which was made of pressed powders, was attached to the mold cavity surface. The compact was made from titanium and carbon powders $(44 \mu \mathrm{m})$ in amount of $50 \mathrm{wt} . \%$ and a moderator which balanced the weight.

The chemical composition of the moderator corresponded to the composition of Hadfield cast steel, which was selected as the base material. The strict chemical composition of the compact is shown in Table 1.

Table 1

Chemical composition of compact, wt.\%

\begin{tabular}{ccccc}
\hline $\mathbf{C}$ & $\mathbf{T i}$ & $\mathbf{F e}$ & $\mathbf{M n}$ & $\mathbf{S i}$ \\
\hline 9.1 & 41.5 & 38.7 & 10.5 & 0.3 \\
\hline
\end{tabular}

The diameter of the compact was $30 \mathrm{~mm}$, and the height was $10 \mathrm{~mm}$. Compaction was carried out at a pressure of $450 \mathrm{MPa}$. In the compact, 3 thermocouples type $\mathrm{S}$ were installed with the step equal to $5 \mathrm{~mm}$. Positions of the thermocouples are presented in Figure 2. All of the thermocouples were placed in quartz tubes with an external diameter of $3 \mathrm{~mm} .10 \mathrm{~mm}$ wide plates of insulating material, made from aluminum silicates fibers, were mounted on the top and bottom sides of compact to minimize the vertical component of the heat flow in the LCR region.

Temperature measurement was performed with a multichannel Agilent 34970A data logger. The device performed temperature measurements at uniform time intervals of $288 \mathrm{~ms}$. The obtained time step for recording measurements for each of the four thermocouples was $1.153 \mathrm{~ms}$ respectively.

The evaluation of the radial temperature gradient in the cylindrical compact was calculated using the central difference scheme for points located $2.5 \mathrm{~mm}$ (on the base of temperature measurement in the points R0 and R5) and $7.5 \mathrm{~mm}$ (on the base of temperature measurement in the points R5 and R10) from the compact axis of symmetry. In the center point of the compact, the constant temperature gradient equal to $0 \mathrm{~K} / \mathrm{m}$ was assumed due to the axial symmetry of this element.

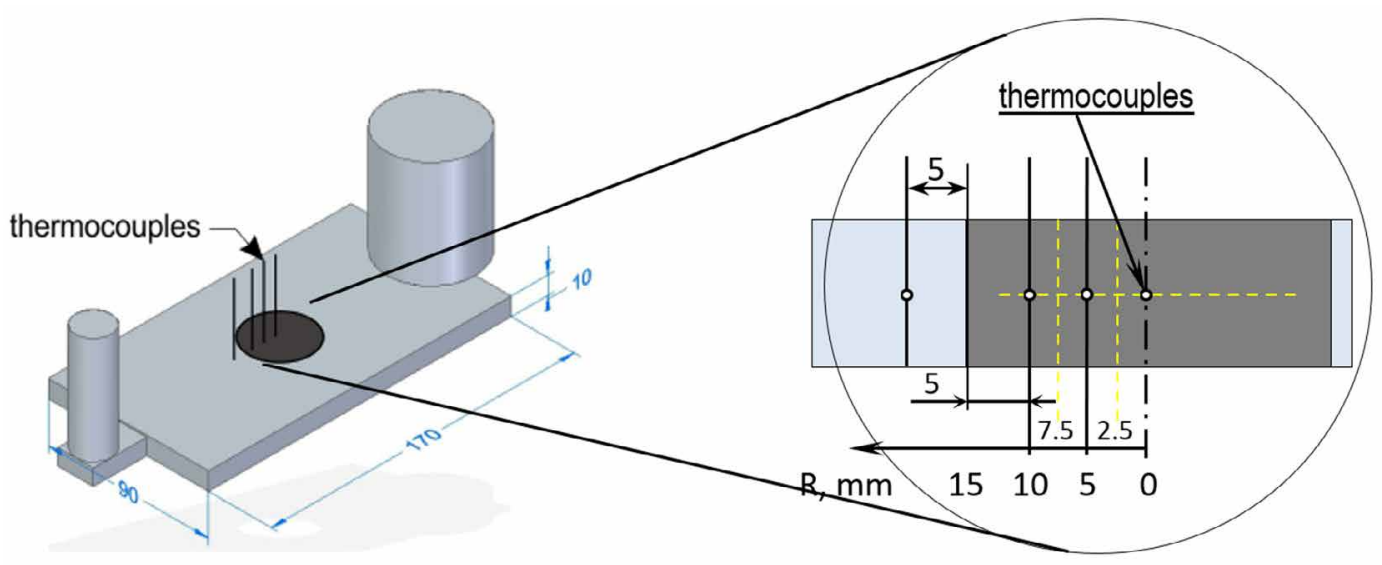

Fig. 2. Pattern of experimental casting 


\section{RESULTS AND DISCUSSION}

The results of temperature measurements are shown in Figure 3.

a)

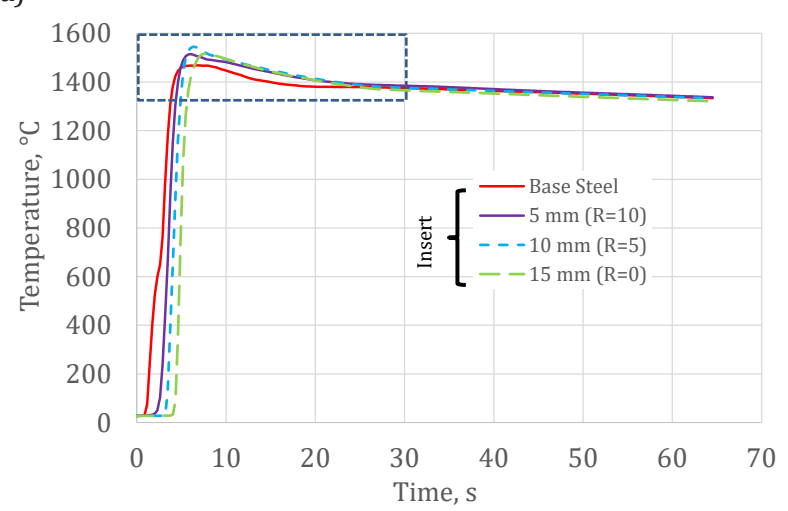

b)

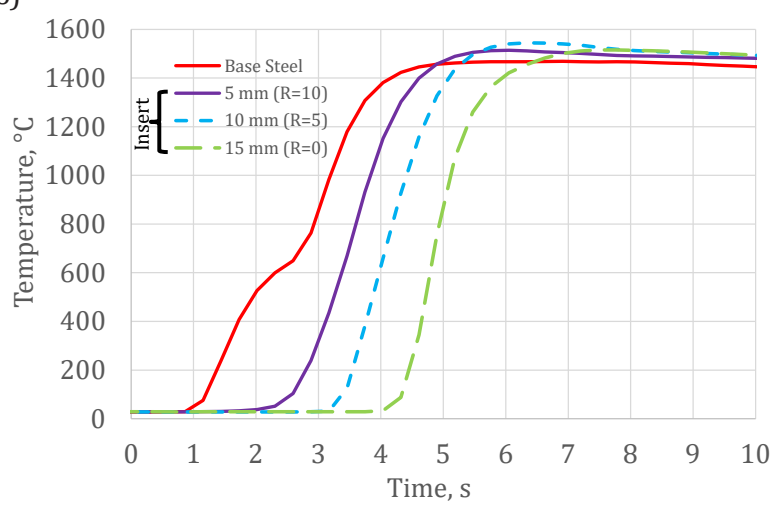

c)

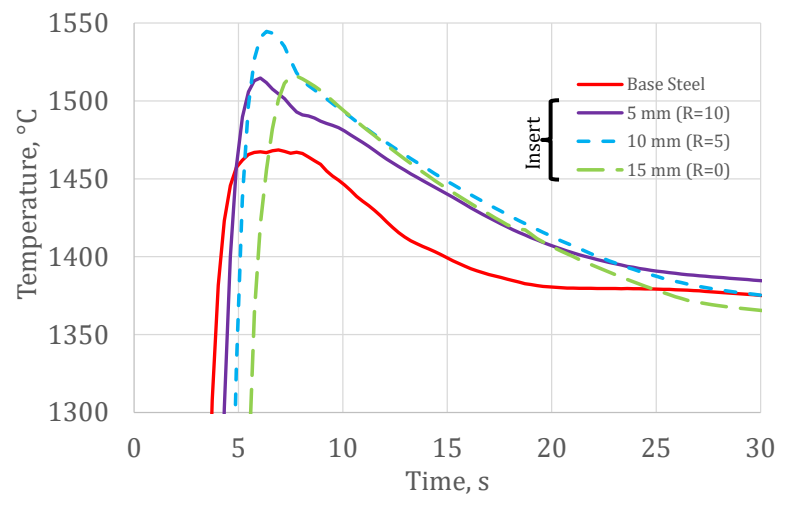

Fig. 3. Temperature changes in the measurement points in the casting and the LCR zone: a) general view; b) temperature increase in the initial period; c) enlargement of the area marked in Figure 3a

Temperature recorded by individual thermocouples rises during the mold filling period. It can be noted that, when the temperature in the liquid steel area exceeds approx. $600^{\circ} \mathrm{C}$, the rate of temperature increase in the liquid steel area grows significantly. This is confirmed by the presence of a local minimum on the temperature change rate curve in the base steel area between 2 and 3 seconds of the process (see Fig. 4). This effect can be linked with the fact that the time instant of the exothermic SHS reaction starts in the neighboring compact. It is noteworthy that the relatively low starting temperature of titanium carbide synthesis indicates the very good wettability of components by liquid steel.

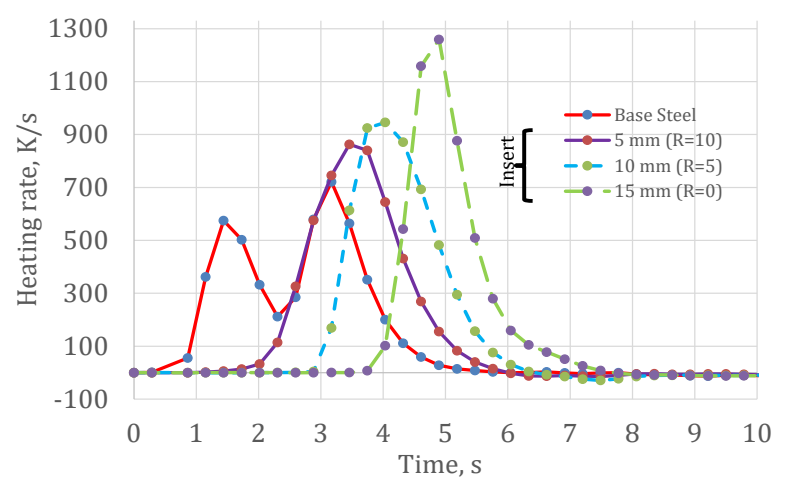

Fig. 4. The rate of temperature changes temperature during mold cavity pouring and SHS reaction of LCR formation in the base casting and in the synthesis zone

The maximum temperature recorded in the area of the synthesis is over $50 \mathrm{~K}$ higher than that recorded in the base casting area (Fig. 3c). The difference between the temperature in the LCR zone and in the casting area disappears after about 25-30 s from the start of mold filling (Fig. 3c).

The diagram of temperature gradient change, calculated on the basis of the recorded temperature measurements, is shown in Figure 5. A change of temperature gradient vector sense in point $7.5 \mathrm{~mm}$ from the compact center after approximately $5.5 \mathrm{~s}$ means that the direction of the heat flux changes from that moment on. If the central part of the compact was initially heated by the steel poured into the mold cavity, and the SHS reaction heat from the outer layer of the compact itself, then from this point of time the temperature in the axis of symmetry of the compact becomes higher, and the central part of the compact is itself cooled through its external layers and the adjacent casting material.

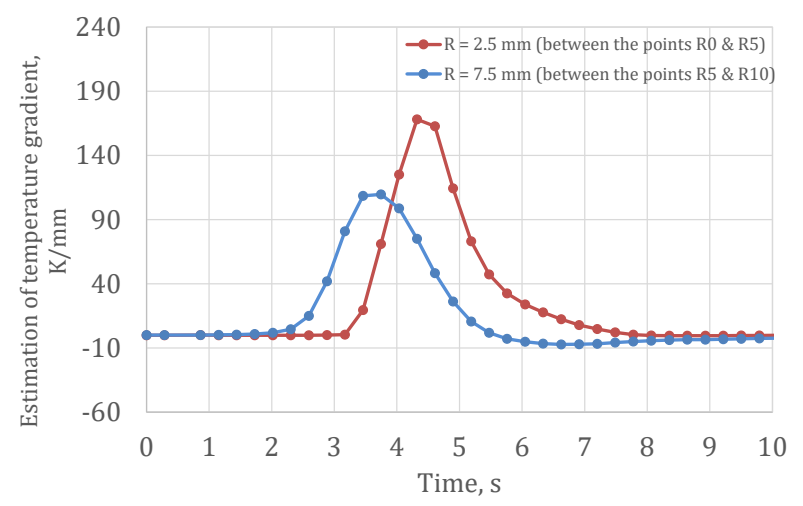

Fig. 5. Evaluation of axial temperature gradient changes in the insert by means of the central difference scheme

The axial symmetry of the compact is taken into account by the calculation of the Laplace operator numerical estimation for the temperature field. The following equation was used in this case:

$$
\frac{\partial^{2} T}{\partial x^{2}} \cong \frac{\left.r_{2} \operatorname{grad} T\right|_{r_{2}}-\left.r_{1} \operatorname{grad} T\right|_{r_{1}}}{r_{2}^{2}-r_{1}^{2}}
$$


The results of this assessment are shown in Figure 6.

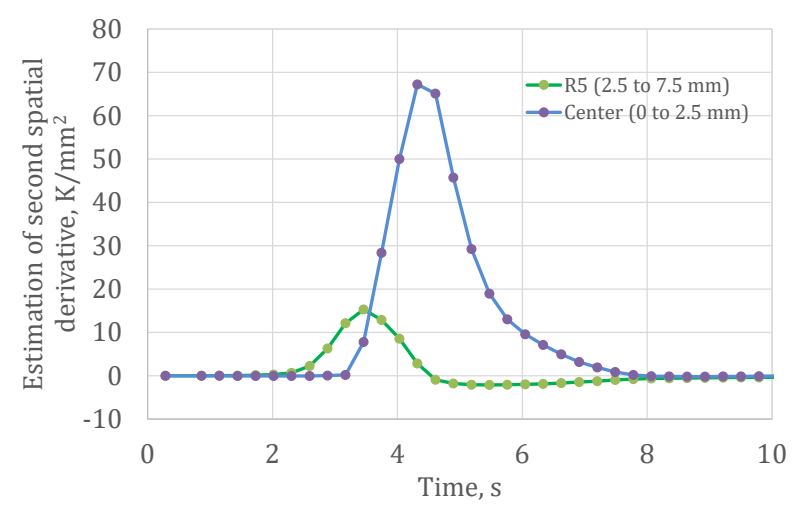

Fig. 6. Laplace operator numerical evaluation for temperature in the insert zone

The measure of the SHS reaction rate $\dot{q}$ is calculated using Equation (3) based on the data presented in Figures 4 and 6. The results of the calculation are presented in Figure 7. The history of the degree of transformation changes calculated from Equation (7) are presented in Figure 8.

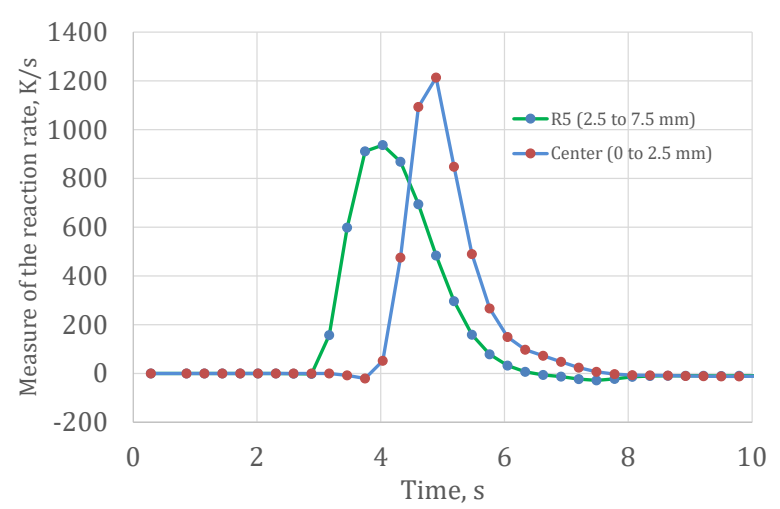

Fig. 7. SHS reaction kinetics of the Ti carbides synthesis in the LCR zone

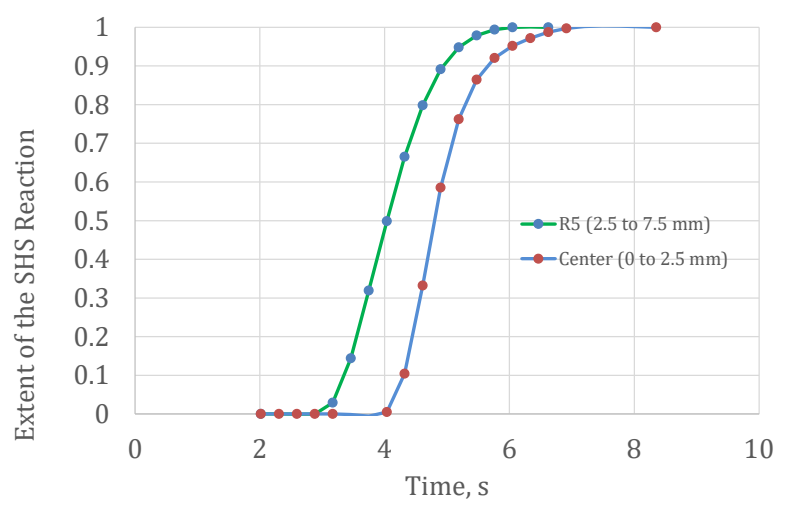

Fig. 8. SHS reaction of TiC completion level in the LCR zone

As it follows from the obtained results, the FTA method may be used for the estimation of the in situ SHS exothermic reaction rate in the case of LCR zone formation in the casting. Calculations were performed on the basis of the measurement results for a compact with a cylindrical symmetry $30 \mathrm{~mm}$ in diameter prepared from the raw materials for
SHS. The estimation results characterize local volumes. In the two cylindrical zones of the compact, the same reaction completion time equal to $3 \mathrm{~s}$ was obtained. In the cylindrical layer within radius boundaries of 7.5 to $2.5 \mathrm{~mm}$, the reaction starts at the time instant of $3 \mathrm{~s}$. In the cylindrical zone with a radius of $2.5 \mathrm{~mm}$, the reaction starts $1 \mathrm{~s}$ later. These results show that the synthesis rate of titanium carbide in substrates for LCR production is very high. The velocity of the reaction front migration from one of the above volumes to the other is nearly $5 \mathrm{~mm} / \mathrm{s}$.

\section{CONCLUSIONS}

In this paper SHS of titanium carbide in cast steel casting was investigated experimentally and numerically. The following conclusion have been reached:

1. Temperature measurements were carried out in the molded compact prepared from titanium and carbon powders $(44 \mu \mathrm{m})$ in amount of $50 \mathrm{wt} . \%$ and moderator (Hadfield steel) during the filling of the mold cavity and in situ SHS reaction of carbide synthesis in the steel casting.

2. The Fourier method of thermal analysis was used to estimate the kinetics of the in situ exothermic SHS reaction of carbides synthesis in the two cylindrical volumes of the LCR zone.

3. The time of the SHS in situ reaction completion during LCR zone formation in the steel casting, as estimated by the FTA method, is nearly $3 \mathrm{~s}$. The velocity of the synthesis reaction front migration is estimated at $5 \mathrm{~mm} / \mathrm{s}$.

\section{REFERENCES}

[1] Olejnik E., Szymański Ł., Tokarski T., Opitek B. \& Kurtyka P. (2019). Local composite reinforcements of TiC/FeMn type obtained in situ in steel castings. Archives of Civil and Mechanical Engineering, 19(4), 997-1005.

[2] Olejnik E., Szymański Ł., Batóg P., Tokarski T. \& Kurtyka P. (2020). TiC-FeCr local composite reinforcements obtained in situ in steel casting. Journal of Materials Processing Technology, 275(1), 116157.

[3] Olejnik E., Tokarski T., Sikora G., Sobula S., Maziarz W., Szymański Ł. \& Grabowska B. (2019). The Effect of Fe Addition on Fragmentation Phenomena, Macrostructure, Microstructure, and Hardness of TiC-Fe Local Reinforcements Fabricated in situ in Steel Casting. Metallurgical and Materials Transactions A, Physical Metallurgy and Materials, 50A(2), 975-986.

[4] Sobula S., Olejnik E., Sikora G. \& Tokarski T. Strefy kompozytowe uzyskane metodą syntezy in situ w staliwie. Konferencja naukowa „Staliwo - nowe wyzwania przemysłowe”, 18-19 April 2013, Kraków.

[5] Ignaszak Z. \& Popieralski P. (2003). Właściwości termofizyczne otulin izolacyjno-egzotermicznych oznaczane metodą zagadnień odwrotnych. Archiwum Odlewnictwa, 3(9), 209-220.

[6] Ignaszak Z. \& Prunier J.-B. (2017). Innovative Laboratory Procedure to Estimate Thermophysical Parameters of Iso-exo Sleeves. Archives of Foundry Engineering, 17(1), 67-72.

[7] Fraś E., Kapturkiewicz W., Burbelko A. \& Lopez H.F. (1993). A New Concept in Thermal Analysis of Castings. AFS Transactions, 101, 505-511. 
[8] Fraś E., Kapturkiewicz W., Burbelko A. \& Lopez H.F. (1997). Numerical simulation and Fourier thermal analysis of solidification kinetics in high-carbon Fe-C alloys. Metallurgical and Materials Transactions B, 28(1), 115-123. Doi: 10.1007/ s11663-997-0134-z

[9] Emadi D. \& Whiting L.V. (2002). Determination of Solidification Characteristics of Al-Si Alloys by Thermal Analysis. AFS Transactions, 110, 285-296.

[10] Gonzalez-Rivera C., Campillo B., Castro M., Herrera M. \& Juarez-Islas J.A. (2000). On the Local Microstructural Characteristics Observed in Sand Cast Al-Si Alloys. Materials Science and Engineering: A, A279, 149-159.

[11] Gonzalez-Rivera C., Cruz H., Garcia A. \& Juarez-Islas J.A. (1999). The Effect of Heat Transfer on Local Solidification Kinetics of Eutectic Al-Si Cast Alloy. Journal of Materials Engineering and Performance, 8(1), 103-110.

[12] Djurdjevic M.B., Odanovic Z. \& Talijan N. (2011). Characterization of the Solidification Path of AlSi5Cu (1-4 wt.\%) Alloys Using Cooling Curve Analysis. JOM, 63(11), 51-57.

[13] Moraru L. (2000). Fourier Thermal Analysis of Solidification Kinetics in Molten Aluminium and in Presence of Ultrasonic Field. Czechoslovak Journal of Physics, 50(10), 1125-1132.
[14] Piasentini F., Bonollo F. \& Tiziani A. (2005). Fourier thermal analysis applied to sodium eutectic modification of an ALSI7 alloy. Metallurgical Science and Technology, 23(2), 11-20.

[15] Han Z., Pan H., Li Y., Luo A.A. \& Sachdev A.K. (2015). Study on Pressurized Solidification Behavior and Microstructure Characteristics of Squeeze Casting Magnesium Alloy AZ91D. Metallurgical and Materials Transactions B, 46(1), 328-336.

[16] Cruz H., Ramirez-Argaez M., Juarez A., Garcia A. \& González-Rivera C. (2009). Fourier Thermal Analysis of the Eutectic Formed in Pb-Sn Alloys. Journal of Materials Engineering and Performance, 18(5), 441-445.

[17] Baez J.C., Gonzalez C., Chavez M.R., Castro M. \& Juarez J. (2004). Fourier Thermal Analysis of the Solidification Kinetics in A356/SiC $\mathrm{S}_{\mathrm{p}}$ Cast Composites. Journal of Materials Processing Technology, 153(SI), 531-536.

[18] Svidro J.T., Dioszegi A. \& Toth J. (2014). The Novel Application of Fourier Thermal Analysis in Foundry Technologies. Journal of Thermal Analysis and Calorimetry, 115(1), 331-338.

[19] Toth J., Svidro J.T., Dioszegi A. \& Stevenson D. (2016). Heat Absorption Capacity and Binder Degradation Characteristics of 3D Printed Cores Investigated by Inverse Fourier Thermal Analysis. International Journal of Metalcasting, 10(3), 306-314. 